\section{La construcción de conocimiento y la integralidad de funciones en la agenda actual de la extensión universitaria}

\author{
Verónica Zabaleta
}

Comisión de Investigaciones Científicas de la Provincia de Buenos Aires. Universidad Nacional de La Plata, Argentina.

veronicazabaleta@gmail.com
Investigación y extensión universitaria / Perspectivas
RECEPCIÓN: 29/06/18

ACEPTACIÓN FINAL: 26/07/18

\section{Resumen}

El presente trabajo se propone abordar la temática de la construcción de conocimiento en relación con las prácticas extensionistas y vincular la relevancia de este tema con dos cuestiones principales. Por un lado, con la necesidad de revalorizar saberes no académicos si la universidad pretende establecer un diálogo con la comunidad a la que pertenece. Por otro lado, con el carácter estructurante del conocimiento no solo para la extensión sino también para el resto de las funciones sustantivas de la universidad. De aquí se deriva el análisis de la integralidad como tema de la agenda de la extensión universitaria y de lo que se ha denominado la "Segunda Reforma Universitaria". El problema epistemológico que el trabajo aborda se articula, asimismo, con cuestiones de índole ética y política.

Palabras clave: extensión, universidad, conocimiento, integralidad, ética
The construction of knowledge and the integrality of functions in the current agenda of university extension

\section{Abstract}

The present work aims to address the issue of knowledge construction in relation to extension practices, linking the relevance of this topic with two main issues. On the one hand, with the need to revalue nonacademic knowledge if the University intends to establish a dialogue with the community to which it belongs. On the other hand, with the structuring character of knowledge, not only for extension, but also for the rest of the substantive functions of the University. From this, the analysis of integrality is derived not only as theme of the university extension agenda but also of what has been called the "Second University Reform". The epistemological problem that the work addresses is also articulated with questions of an ethical and political nature.

Keywords: extension, university, knowledge, integrality, ethics
A construção de conhecimento e a integralidade de funções na agenda atual da extensão universitária

\section{Resumo}

No seguinte trabalho propõe-se abordar a temática da construção de conhecimento relacionada às práticas extensionistas, vinculando a relevância deste tema com dois assuntos principais. Por um lado, com a necessidade de revalorizar saberes nãoacadêmicos caso a Universidade pretenda estabelecer um diálogo com a comunidade à que pertence. Por outro, com o caráter estruturante do conhecimento, não somente para a extensão, mas também para as outras funções essenciais da Universidade. A partir disso deriva-se a análise da integralidade não só como tema da agenda da extensão universitária, mas também do que tem sido denominada "Segunda Reforma Universitária". O problema epistemológico que este trabalho aborda articula-se, ao mesmo tempo, com questões de índole ética e política

Palavras-chave: extensão, universidade, conhecimento, integralidade, ética

\title{
$-$
}

Para citación de este artículo: Zabaleta, V. (2018). La construcción de conocimiento y la integralidad de funciones en la agenda actual de la extensión universitaria. +E: Revista de Extensión Universitaria, 8(8), enero-junio, 12-25. doi: 10.14409/extensión.v8i8.Ene-Jun.7710. 


\section{Introducción}

Este trabajo se propone analizar dos temáticas relevantes de la agenda actual de la extensión universitaria (en adelante EU) que, si bien tienen su especificidad, pueden abordarse de modo articulado.

Una primera cuestión a considerar es por qué la temática de la construcción de saberes se torna central cuando se aborda la EU, es decir: ¿por qué es importante preguntarse sobre el conocimiento y su construcción en relación con la extensión?

En principio podría plantearse que la incorporación de este tema a la agenda contemporánea de la EU se vincula a una necesidad de revalorizar conocimientos y saberes no estrictamente universitarios. Esto es, el motivo no es visibilizar la importancia de lo que la universidad como institución produce en términos de conocimientos, sino más bien realizar una especie de autocrítica relativa a la histórica tendencia del discurso universitario a situarse por encima de otros contextos de producción de conocimiento. En este sentido, rápidamente se establece una dicotomía entre el conocimiento científico, académico, experto, que se suele relacionar a la universidad y a la investigación, y el conocimiento de sentido común, empíri$\mathrm{co}$, o los saberes populares, ligados con lo que, genéricamente, se denomina la comunidad o los destinatarios de las prácticas extensionistas.

Podría decirse entonces que uno de los temas de la agenda contemporánea de la EU se vincula precisamente a su delimitación como práctica de encuentro, diálogo, intercambio, negociación y/o articulación de saberes. Es decir que la extensión ubica en su propia agenda estos rasgos que parecen definirla, abriéndolos a la reflexión y al debate tal vez mucho más de lo que parecen hacerlo otras prácticas universitarias como la docencia y la investigación.

Sin embargo, no es por lo único que este tema se torna significativo, en tanto que el conocimiento y su construcción no pueden considerarse solamente un eje de la agenda de la extensión, sino que son el elemento estructurante de las funciones sustantivas de la universidad. De allí el interés que reviste su abordaje.

Permiten repensar la relación de la universidad con otros contextos no universitarios pero también reflexionar sobre las funciones centrales de esta institución. Y esto posibilita articular la temática de la construcción de saberes con otro eje central de la agenda de la extensión que es la denominada integralidad de las funciones universitarias.

En este escrito se partirá de analizar qué se entiende por EU, retomando documentos producidos en el contexto de la Universidad Nacional de La Plata, como también otros aportes que enriquecen su conceptualización. Luego se analizará la temática del diálogo de saberes, considerando al conocimiento como estructurante de las funciones sustantivas de la universidad, y se propondrá una reflexión de índole epistemológica. Finalmente, se abordará la temática de la integralidad de funciones.

\section{Algunas consideraciones acerca de la extensión en el contexto universitario}

Partiremos, en el análisis a realizar en este trabajo, de las definiciones y precisiones que establece el Estatuto, el Plan Estratégico de Gestión (2014-2018) y el Proyecto Institucional (2018-2022) "Pensar la Universidad" (Tauber, 2016), de la Universidad Nacional de La Plata (UNLP), respecto de la extensión universitaria. 
El Estatuto reconoce a la EU como una de sus funciones primordiales a la par de la investigación y la extensión. Plantea que la EU busca construir respuestas a problemas sociales, fundamentalmente de aquellos sectores que pueden considerarse vulnerables por no tener sus derechos esenciales garantizados. La define como el principal medio de la UNLP para lograr su función social, contribuyendo a la reconstrucción del tejido social, al desarrollo económico sustentable y al fortalecimiento de la identidad cultural.

En su artículo $17^{\circ}$, la define como:

"un proceso educativo no formal de doble vía, planificada de acuerdo a intereses y necesidades de la sociedad, cuyos propósitos deben contribuir a la solución de las más diversas problemáticas sociales, la toma de decisiones y la formación de opinión, con el objeto de generar conocimiento a través de un proceso de integración con el medio y contribuir al desarrollo social" (2008:9).

Si sintetizamos los aspectos principales contenidos en estas definiciones, puede decirse entonces que la EU:

- es una de las funciones que cumple la UNLP conjuntamente con otras dos: la docencia y la investigación;

- es una práctica de resolución de problemas, principalmente de sectores sociales cuyos derechos han sido vulnerados, sin que medie lucro alguno entre los involucrados;

- es el principal medio con que cuenta la Universidad para lograr su función social vinculada a la reconstrucción del tejido social, a la sustentabilidad y al fortalecimiento de la identidad cultural;

- es un proceso educativo no formal de doble vía que implica al mismo tiempo la resolución de problemas sociales y la producción contextualizada de conocimiento.

En tanto, el Plan Estratégico de Gestión de la UNLP (2014-2018) delimita seis líneas estratégicas que atienden a la Enseñanza, la Investigación, la Extensión, el Arte y la Cultura, las Relaciones Institucionales y la Gestión y Administración Institucional.

La tercera línea estratégica, ligada a la EU, plantea como objetivo general:

"Desarrollar y consolidar prácticas extensionistas que integren la universidad, en tanto institución del Estado Nacional, con la comunidad de la cual forma parte, promoviendo la articulación, la comunicación, la expresión y el intercambio de saberes que contribuyan en la resolución de las diversas problemáticas sociales, desde una perspectiva integral e interdisciplinaria". (51)

Luego se derivan de este objetivo general, un producto, objetivos específicos, resultados esperados, programas generales, programas específicos, subprogramas, subprogramas operativos y proyectos.

Si se analizan el objetivo general y los específicos propuestos, puede señalarse que el Plan Estratégico destaca la importancia de:

- la articulación, comunicación, expresión e intercambio de saberes;

- una perspectiva interdisciplinaria;

- la integración de las actividades extensionistas con las actividades docentes y de investigación; 
- la integración de la Universidad con la comunidad de la que forma parte, la articulación con organismos gubernamentales y no gubernamentales;

- la participación de la Universidad en la definición de políticas públicas;

- la inserción curricular de la EU;

- la participación en redes de EU;

- la difusión y producción de contenidos en relación con las prácticas extensionistas.

Asimismo, el Plan Estratégico incluye un análisis de fortalezas, oportunidades, debilidades y amenazas (FODA) referido a la EU.

Se señalan de modo explícito, y en el marco de la "fortalezas", las acciones tendientes a la jerarquización de la EU que la institución viene poniendo en marcha y se agrega una idea interesante, que debe ser considerada con cautela, referida a la "obligatoriedad" de la EU. Aquí resulta importante señalar que dicha obligatoriedad recae sobre la Universidad como institución del Estado y no, necesariamente, en los actores individuales que la integran. Si pensamos, por ejemplo, en la planta docente, solo el $11 \%$ cuenta con dedicación exclusiva, el $16 \%$ con semidedicación, y el $73 \%$ restante desempeña funciones exclusivamente docente con dedicaciones simples. Además, el Estatuto prevé que aquellos que cuenten con mayores dedicaciones podrán desempeñar tareas docentes y al menos una de las dos las actividades: investigación y/o extensión. Por lo tanto, el porcentaje de docentes que podrían estar "obligados" a realizar tareas extensionistas es igual o menor al $27 \%$ de la planta docente total de la UNLP.

Entre las debilidades se señalan la escasa sistematización y conceptualización de las experiencias, dificultades vinculadas a la formación de los extensionistas y la exigua articulación con proyectos de investigación.

En clara continuidad con lo desarrollado, el Proyecto Institucional 2018-2022 señala que la extensión:

"Pasó de mero integrante en el discurso histórico de las funciones básicas de la universidad, junto a la enseñanza y la investigación, a ser protagonista activa de procesos sociales asociados a la formación de estudiantes y docentes, con un fuerte vínculo que la comunidad reconoce cada vez con más claridad, y se transformó en referente en el tema entre las universidades del país y la región". (Tauber, 2016:43)

Con el objeto de enriquecer lo que se ha retomado de los documentos de la UNLP, pueden considerarse también otros aportes relativos a qué es la EU y cuál es su valor que están en sintonía con lo ya desarrollado.

Arocena señala que existen diferentes puntos de vista sobre lo que es o lo que debería ser la extensión. Pero lejos de considerar esto un rasgo negativo, sostiene que "ello no es perjudicial, sino todo lo contrario, porque en toda actividad humana relevante el eventual dominio de un 'pensamiento único' adormece, embota, rutiniza y empobrece. La Universidad tiene que ser ámbito de permanente y fermental debate" (2013:10).

Apunta la siguiente definición de EU:

"Concebimos a la extensión universitaria como el conjunto de actividades de colaboración entre actores universitarios y no universitarios, en formas tales que todos los actores involucrados aportan sus respectivos saberes y aprenden en un proceso interactivo orien- 
tado a la expansión de la cultura y a la utilización socialmente valiosa del conocimiento con prioridad a los sectores más postergados" (Arocena:11)

Esta definición es relevante para este trabajo porque pone en primer plano las relaciones de colaboración, del intercambio de saberes, el aprendizaje y la expansión de la cultura.

Boaventura de Sousa Santos (2007), en concordancia con lo que se denomina la misión social de la UNLP, señala que las actividades de extensión deben tener como objetivo prioritario, refrendado democráticamente al interior de la universidad, el apoyo solidario para la resolución de los problemas de exclusión y la discriminación sociales, de tal modo que se dé la voz a los grupos excluidos y discriminados.

Menéndez y Tarabella (2016), por su parte, delimitan cinco dimensiones de la EU que sintetizan aspectos relevantes:

- dimensión institucional, académica, de la EU, que se vincula a su reconocimiento como una de las funciones sustantivas de la Universidad implicada en: a) la construcción y apropiación de conocimiento, b) el fortalecimiento de las políticas públicas y de la democracia; - dimensión pedagógica, que es la que potencia la construcción de nuevas formas de enseñar y de aprender;

- dimensión social, que reconoce a la Universidad como una institución social situada en un contexto temporal y espacial definido y vincula sus prácticas a la inclusión social;

- dimensión comunicacional, que sitúa a la Universidad en un campo de relaciones sociales diverso e implicada en temas de interés público;

- dimensión política, en tanto la EU interpela necesariamente a las políticas públicas y construye su agenda sobre la base de la definición de cuestiones sociales relevantes que requieren ser repensadas y transformadas.

Si bien se reconoce la importancia de todas estas dimensiones, el presente trabajo, por su temática, se centra fundamentalmente en las dimensiones pedagógica y en la comunicacional.

\section{Diálogo de saberes y extensión universitaria}

La concepción presentada sobre la EU parece ubicarla en un lugar estratégico en relación con la producción de conocimiento al interior de las disciplinas, la formación de profesionales y la función social de la Universidad como institución del Estado.

Menéndez (2015) plantea una idea que servirá en este trabajo como punto de partida: el conocimiento estructura las funciones sustantivas de la Universidad. Es decir que el modo en que el conocimiento se produce, se valida y se socializa, constituye un eje central de la universidad como institución y, por lo tanto, atraviesa el conjunto de sus funciones: docencia (grado y posgrado), investigación y extensión. Esto implica que, si nos preguntamos por la construcción de conocimiento en el ámbito de las prácticas extensionistas y caracterizamos a ese proceso como de diálogo, encuentro, estaremos inevitablemente asumiendo una posición epistemológica que "irradia" sus efectos hacia las otras funciones sustantivas y a los modos posibles de pensar sus relaciones. Esto también se relaciona con una manera peculiar de entender la integralidad de las funciones de la Universidad, lo que se desarrollará en el apartado siguiente. 
La temática de la construcción del conocimiento en relación con la EU concebida como práctica social puede situarse en un contexto más amplio vinculado al debate respecto del positivismo en el campo de las ciencias sociales. Dicho debate se centra en la crítica a tres de sus ejes fundamentales: el reduccionismo, el determinismo y el dualismo.

Dentro del campo de la Sociología, Sousa Santos (2006) realiza aportes que pueden resultar significativos en el análisis de los saberes implicados en la EU. En su intento de "reinventar la emancipación social" propone tres dimensiones de análisis: la epistemológica, la teórica y la política. En este trabajo se retoman fundamentalmente desarrollos ligados a la primera dimensión.

Según este último autor, el mundo se caracteriza por una diversidad epistemológica inagotable y las ciencias sociales sostienen una racionalidad que, en contraposición, tiende a ser reduccionista e indolente, desconoce dicha diversidad y "desperdicia" la experiencia. Caracteriza esta racionalidad haciendo referencia a dos de sus manifestaciones: la razón metonímica y la razón proléptica.

En el primer caso, se alude a un tipo de racionalidad que toma la parte por el todo, en tanto concibe a la totalidad como constituida por partes homogéneas. Al hacer esto contrae, disminuye el presente e invisibiliza aspectos de la realidad y la experiencia. Una idea relacionada con ello es la de simetría dicotómica, que oculta siempre una jerarquía. Es decir que nuestra racionalidad define dicotomías que parecen simétricas aunque ocultan diferencias y jerarquías. Nuestra racionalidad se basa en la transformación de lo real sin comprenderlo. Y la transformación sin comprensión lleva a situaciones de desastre. Así, para combatir la razón metonímica propone una sociología de las ausencias, que implica considerar que lo que no existe en nuestra sociedad es producido activamente como no existente. Hay cinco modos de producción de ausencias en nuestra racionalidad occidental que las ciencias sociales comparten: la monocultura del saber y del rigor, la monocultura del tiempo lineal, la monocultura de la naturalización de las diferencias que ocultan jerarquías, la monocultura de la escala dominante y la monocultura del productivismo capitalista.

La monocultura del saber y del rigor se sostiene en la idea de que el único saber riguroso es el científico y, por lo tanto, otros conocimientos no tienen igual validez ni rigor. Produce lo que se denomina "epistemicidio", que es la muerte de conocimientos alternativos. Esta idea resulta central en el campo de la EU en tanto uno de sus caracteres constitutivos es el vínculo entre agentes que pertenecen a la Universidad como institución que produce y valida sus propios saberes reconocidos como de carácter científico y otros agentes no universitarios, que no se reconocen a sí mismos (y tampoco la Universidad lo hace) como componentes del vínculo en virtud de la mencionada pertenencia (aunque puedan ser universitarios). Esto plantea necesariamente una relación de asimetría puesto que el vínculo se funda en el reconocimiento de la diferencia entre lugares. Y lo que se ha reconocido como problemático es la transformación de esa asimetría basada en la diferencia en jerarquías sostenidas en la desigualdad; esto es, que lo diferente es inferior, descartable y, por lo tanto, no amerita el diálogo.

En este sentido, la monocultura de la naturalización de las diferencias que ocultan jerarquías es otra característica de la racionalidad perezosa occidental que no sabe pensar diferencias con igualdad; las diferencias son siempre desiguales. Por consiguiente, el tercer modo de producir ausencia que conceptualiza Sousa Santos es "inferiorizar", que es una manera descalificada de alternativa a lo hegemónico, precisamente, por ser inferior. 
La sociología de las ausencias que propone el autor es una sociología insurgente que sustituye las monoculturas por las ecologías que son también cinco: ecología de saberes, ecología de las temporalidades, ecología del reconocimiento, ecología de la transescala y ecología de las productividades.

La ecología de saberes es la posibilidad de que la ciencia entre, no como monocultura, sino como parte de una ecología más amplia de saberes, donde el saber científico pueda dialogar con el saber laico, con el popular, con el de los indígenas, con el de las poblaciones urbanas marginales, con el campesino. Pero esto no significa que todo vale lo mismo. Propone una concepción pragmática del saber en tanto considera el tipo de intervención en la realidad que el mismo produce:

"No hay duda de que para llevar al hombre o a la mujer a la luna no hay conocimiento mejor que el científico; el problema es que también sabemos hoy que para preservar la biodiversidad, de nada sirve la ciencia moderna. Al contrario, la destruye. Porque lo que ha conservado y mantenido la biodiversidad son los conocimientos indígenas y campesinos". (Sousa Santos, 2006:27)

La denominada ecología del reconocimiento implica un procedimiento de descolonización de nuestras mentes para poder producir algo que distinga, en una diferencia, lo que es resultado de la jerarquía y lo que no lo es. Sousa Santos considera que solo se deben aceptar las diferencias que queden después de que las jerarquías sean desechadas.

La razón proléptica expande infinitamente el futuro, y la crítica a esta es realizada por otra sociología insurgente, que es la sociología de las emergencias. En este caso, se trata de intentar contraer el futuro: sustituirlo como infinito por un futuro concreto, de utopías realistas, suficientemente utópicas como para desafiar a la realidad que existe pero realistas como para no ser descartadas fácilmente. Lo que no existe pero está emergiendo. Entre la nada y el todo, propone el "todavía no". No es un futuro abstracto, es un futuro del cual tenemos pistas y señales; tenemos gente involucrada, que dedica su vida $-\mathrm{y}$ muere muchas vecespor esas iniciativas.

La sociología de las ausencias y de las emergencias nos va a confrontar con una realidad más rica, heterogénea y fragmentada. ¿Cómo producir sentido? Propone un procedimiento que denomina "de traducción": traducir saberes en otros saberes, traducir prácticas y sujetos de unos a otros, es buscar inteligibilidad sin "canibalización", sin homogeneización. Hay que crear inteligibilidad sin destruir la diversidad. Sostiene que hay muchos lenguajes para hablar de la dignidad humana, para hablar de un futuro mejor, de una sociedad más justa. La Epistemología que propone se basa en la idea de que no hay justicia social global sin justicia cognitiva global; o sea, sin justicia entre los conocimientos.

Sousa Santos (2007) considera que se puede hacer ecología de saberes dentro de la universidad. Mientras que la extensión convencional es llevar la universidad hacia afuera, la ecología de saberes es traer otros conocimientos hacia adentro de la universidad. Esto no implica que los saberes universitarios construidos en el marco de la investigación pierdan objetividad ya que existe un rico desarrollo epistemológico y metodológico en el campo de las ciencias que intenta producir conocimientos rigurosos que son, por otra parte, también diversos. Es decir que la diversidad no es solo un rasgo de los conocimientos o saberes no científicos. 
La dicotomía entre conocimiento científico y conocimiento cotidiano, u otras dicotomías que intentan explicitar algo similar, necesariamente empobrecen, "contraen" la experiencia.

Sousa Santos (2006) apunta a diferenciar entre objetividad y neutralidad. En lo que respecta a la ciencia, se puede ser objetivo pero no neutro:

"Objetividad, porque poseemos metodologías propias de las ciencias sociales para tener un conocimiento que queremos que sea riguroso y que nos defienda de dogmatismos; y al mismo tiempo, vivimos en sociedades muy injustas en relación a las cuales no podemos ser neutrales. Debemos ser capaces de efectuar esta distinción, que es muy importante". (18)

Esto plantea otra dimensión que resulta central, que es la de la ética, no solo en relación con las prácticas extensionistas sino también respecto del conjunto de las funciones universitarias. La EU pensada como diálogo de saberes supone un principio de reconocimiento del otro en su diversidad que debe considerarse desde una perspectiva ética. Esto transforma no solamente a la EU, que deja de ser transferencia o asistencia técnica, sino a las modalidades que asumen la producción, validación y socialización del conocimiento científico y a los procesos de enseñanza y de aprendizaje en el contexto universitario. Según Cecchi:

"Estas intervenciones conforman un escenario privilegiado para que el proceso de enseñar y de aprender incluya una dimensión distinta. Enseñar y aprender en un contexto real tiene implicancias realmente muy distintivas en la formación de alguien. Esto lo hemos planteado, conversado y escrito; pero enseñar y aprender en contextos reales, en contextos experienciales, amplifica, redimensiona y resignifica el contenido que se pone en juego. El estudiante que aprende en términos reales en el territorio, lo hace de otro modo: aprende más, mejor y con sentido ético". (2015:30)

Maiorana realiza consideraciones interesantes en cuanto a lo que entendemos por diálogo al retomar reflexiones filosóficas occidentales tempranas. Considera que este tuvo un lugar importante para los griegos en relación con el teatro:

"El concepto de diálogo en el teatro se da cuando el primer actor, que se llama protagonista, dialoga con el coro, que es toda la masa. Recién hay diálogo verdadero cuando de la masa se separa un segundo actor, que se llama el antagonista (neutrogonista), y empieza a dialogar con el protagonista. Desde ese momento, el protagonista deja de serlo, porque en realidad es un sujeto que dialoga. $Y$ esto es lo interesante cuando uno dialoga desde un punto de vista pragmático, lingüístico, etc. uno se pone al mismo nivel que el otro, respetando los turnos. Al mismo tiempo debe respetar a ese otro, escucharlo y ser a la vez escuchado, independientemente de la posición que adopte cada uno de los dos dialogantes o dialoguistas. Lo importante es que si uno dialoga, tiene que asumirse como tal, y tiene que asumirse también con humildad, porque el que no se asume con humildad como dialoguista, es en realidad un monologuista" (2015:12)

Estos desarrollos que enfatizan el papel del diálogo y su relación con la ética no deben promover la imagen de que en la EU se establecen vínculos "libres de conflicto". La EU es una práctica de resolución de problemas y, como tal, implica necesariamente el conflicto y la negociación. Desde el momento mismo en que algo de la realidad se construye como 
problema, se lo hace con una determinada perspectiva, y no siempre las perspectivas son compartidas. Y aun si lo son en lo aparente, pueden conllevar interpretaciones diversas y representaciones diferentes de cuáles son las vías adecuadas para su abordaje y/o resolución.

Erausquin (2013), retomando a Ferrero (2012), propone el concepto de ética dialógica, unidad de análisis relevante para el tema que nos ocupa. Toda intervención profesional implica una posición ética, explícita o no. El posicionamiento ético involucra una vigilancia epistémica continua, con rigor teórico y efectividad técnica; pero, más allá de lo epistémico, supone compromiso e implicación con el destino común de la humanidad. La ética dialógica interpela a la responsabilidad social que todos tenemos, apunta al bienestar, al mejoramiento de la vida de todos y a la búsqueda de consensos que no eludan los conflictos.

Esto permite comprender, entonces, que un problema epistemológico como el que aborda este trabajo se articula con cuestiones de índole ética y política.

\section{Extensión universitaria e integralidad de funciones}

Tal como afirma Sousa Santos: "en el siglo XXI solo habrá universidad cuando haya formación de grado y de posgrado, investigación y extensión. Sin cualquiera de estas habrá enseñanza superior pero no habrá universidad" (2007:59).

La integralidad refiere precisamente al modo de pensar el vínculo entre las funciones sustantivas de la universidad asumiendo que son ellas las que le otorgan su especificidad. Es decir, que de la misma manera que se planteaba más arriba un proceso de diálogo de saberes en la relación entre la universidad y otros ámbitos no universitarios, es preciso concebir el acto educativo en la universidad como un proceso de diálogo entre la enseñanza, la investigación y la extensión. Esto no implica el desdibujamiento de la especificidad de cada una de ellas sino la delimitación de la misma a partir de su relación en el contexto de un sistema.

Arocena plantea "extender la extensión" al conjunto de la institución:

"hace falta mostrar que todas las actividades de enseñanza y creación de conocimientos y cultura pueden, de manera bastante natural, vincularse con actividades de extensión en sí mismas valiosas $\mathrm{y}$, además, susceptibles de enriquecer tanto las modalidades educativas como las agendas de investigación". (2013:13)

En un documento de la Universidad de la República (UR Rectorado, 2010, citado en Tommasino y Rodríguez) se especifica qué se puede entender por prácticas integrales:

"a) la integración de los procesos de enseñanza y construcción de conocimientos a experiencias de extensión; b) la perspectiva interdisciplinaria tanto en el nivel epistemológico vinculado a la enseñanza y a la investigación, como en el nivel de la intervención; c) la intencionalidad transformadora de las intervenciones concibiendo a los actores sociales como sujetos protagonistas de dichas transformaciones y no como objeto de intervenciones universitarias (participación comunitaria, diálogo de saberes y ética de la autonomía); d) la concepción integral de los procesos de enseñanza y aprendizaje, tanto en el tratamiento de los contenidos como en las metodologías (ecología de saberes, enseñanza activa, aprendizaje por problemas); e) enfoque territorial e intersectorialidad en el abordaje de las intervenciones". (2013:24). 
Puede verse claramente que la integralidad no constituye una temática "encapsulada" en la agenda de la EU. A partir de problemas que tal vez la EU ayuda a visibilizar, recortar/construir, analizar, se propone una reflexión que se expande a la universidad como institución y que implica al conjunto de sus funciones sustantivas.

En este sentido, Tommasino y Rodríguez agregan:

"un primer movimiento realizado fue pasar de considerar a la extensión como un aspecto importante en el proceso de Reforma Universitaria, a considerarla como el punto de partida para el desarrollo de prácticas integrales, y en consecuencia pensarla como una de las herramientas de transformación de la Universidad. La función de extensión no puede ser entendida como algo aislado, encapsulada en un gueto, sino que debe ponerse en interacción en el acto educativo y formar parte de manera naturalizada en el mismo". (2013:21)

Sutz, por su parte, insta a pensar la integralidad como un espacio de preguntas recíprocas entre la enseñanza, la investigación y la extensión. A partir de asumir una perspectiva histórica, la autora muestra que la extensión como función universitaria aparece reconocida tardíamente hacia fines de siglo XIX y principios de siglo XX, en relación con la transferencia en el ámbito rural o agropecuario. Afirma:

"De este modo la integralidad como un espacio de preguntas recíprocas implica preguntas que la enseñanza le hace a la investigación, que la investigación le hace a la extensión, y que la extensión le realiza a cualquiera de las otras dos funciones. Asimismo todas las funciones se formulan preguntas a sí mismas y a todas las demás". (2013:52)

Otra propuesta que puede constituir un aporte para el abordaje de la temática de la integralidad remite al enfoque de Yrjö Engeström (2001a, 2001b, 2016) referido a la teoría de la actividad. La denominada por el autor como «tercera generación de la teoría de la actividad» propone definir como unidad mínima de análisis la interacción entre dos sistemas de actividad. Plantea cinco principios que pueden contribuir a pensar la interacción entre enseñanza, investigación y extensión como tres sistemas de actividad en interrelación.

El primer principio que señala Engeström (2001b) refiere a que un sistema de actividad colectivo mediado por artefactos y orientado a objetos, considerado en su red de relaciones con otros sistemas de actividad, puede constituirse en la principal unidad de análisis.

Un sistema de actividad humano implica la relación entre múltiples componentes:

- un sujeto, que no se identifica necesariamente con un individuo particular, cuyo accionar se elige como punto de partida del análisis;

- un objeto/objetivo que refiere a aquello que motiva, da sentido y dirección a la actividad, es el "espacio problemático" de la actividad;

- los artefactos mediadores (herramientas y signos), que precisamente median la relación entre el sujeto y el objeto/objetivo de la actividad para la obtención de ciertos resultados; - la comunidad, que incluye individuos y/o subgrupos que comparten el mismo objetivo general;

- la división del trabajo, que refiere tanto a la división horizontal de las tareas como a la división vertical del poder y la posición;

- las reglas, que conprenden las normas, reglamentaciones y convenciones explícitas e implícitas que regulan las acciones y las interacciones del sistema. 
Cada una de las funciones sustantivas de la universidad podría analizarse sobre la base de este modelo concibiéndolas como sistemas humanos de actividad pasibles de ser caracterizados en virtud del modo peculiar en que estos componentes descritos se concretan y se ponen en relación. A su vez, pueden pensarse las relaciones entre enseñanza, investigación y extensión, a partir de la idea de sistemas de actividad en interacción, tal como lo formula la tercera generación de la teoría.

El segundo principio alude a la multiplicidad de voces. Un sistema de actividad no es un sistema homogéneo sino que, por el contrario, es siempre una comunidad de múltiples puntos de vista, tradiciones e intereses. La división del trabajo en una actividad genera posiciones diversas en los participantes, los que llegan a la actividad con su propia historia, al tiempo que el sistema multiplica diferentes niveles y hebras de su historia anclada en sus artefactos, reglas, convenciones.

El tercer principio es la historicidad. Los sistemas de actividad se organizan y se transforman a lo largo del tiempo. Sus problemas y posibilidades solo pueden entenderse en el contexto de su propia historia.

Cada una de las funciones sustantivas tiene una relación histórica diversa respecto de la universidad como institución y, por lo tanto, respecto de las otras funciones. Por ejemplo, el vínculo entre la enseñanza y la investigación se establece históricamente antes que entre estas y la EU. Pero, a su vez, cada una de ellas no constituye sistemas homogéneos de ideas, principios, normas, convenciones, sino que coexisten múltiples tradiciones que se ligan a momentos históricos diferentes, lo que genera contradicciones.

El cuarto principio refiere, precisamente, a las contradicciones internas como fuente del cambio y del desarrollo. Una actividad humana nunca es un sistema estable y armonioso. La idea de "nueva reforma universitaria" y el concepto mismo de integralidad plantean contradicciones al interior del sistema universitario en su conjunto y en relación con cada una de las funciones sustantivas. Algunas preguntas permitirán ejemplificar lo planteado: ¿La enseñanza debe partir de contenidos disciplinares o de problemas? ¿La agenda de trabajo de los investigadores debe estar orientada únicamente a la publicación de artículos en revistas de alto impacto y/o a la resolución de problemas sociales? ¿La extensión universitaria debe entenderse como transferencia del conocimiento producido en el campo científico o se acerca más a una práctica de resolución de problemas socialmente relevantes a partir de la vinculación con el contexto no universitario? ¿Es lo mismo enseñar aquello que se investiga que reproducir un conocimiento construido por otros? ¿Se enseña de la misma manera cuando docente y estudiante vivencian a través de una experiencia aquello que se quiere transmitir que cuando de lo que se trata es de la exposición de un saber ya elaborado anticipadamente? ¿Es lo mismo construir respuestas a problemáticas sociales a partir de los conocimientos y metodologías que aporta la investigación que hacerlo solo desde la práctica y la experiencia personal en un tema determinado? ¿Qué condiciones requieren los cambios para poder producirse? ¿Entre cuáles de los componentes del sistema se producen las contradicciones?

Finalmente, el quinto principio sostiene la posibilidad de transformaciones expansivas en los sistemas de actividad. Estos cambian a través de ciclos largos de transformaciones cualitativas. Una transformación expansiva se logra cuando el objeto y el motivo de la actividad son reconceptualizados para incorporar un horizonte de posibilidades radicalmente más amplio, que en la anterior modalidad de la actividad no estaba contemplado. 


\section{Discusión y conclusiones}

El presente trabajo ha abordado la compleja temática de la construcción de conocimiento en relación con las prácticas extensionistas vinculando la relevancia que este tema ha cobrado con dos cuestiones principales. Por un lado, con la necesidad de revalorizar saberes no académicos si la universidad pretende establecer un diálogo con la comunidad a la que pertenece. Por otro lado, en relación con el carácter estructurante del conocimiento no solo para la extensión sino también para el resto de las funciones sustantivas de la universidad. De aquí se deriva el análisis de la integralidad como tema de la agenda de la EU y de lo que se ha denominado la "Segunda Reforma Universitaria", que "apunta a revitalizar el valor inspirador del ideal latinoamericano de Universidad, aproximándolo a la realidad en las condiciones sociales y culturales de nuestro tiempo" (Arocena, 2013:10).

No existe un concepto único de extensión, si bien muchas de las definiciones que las universidades han elaborado en estatutos y otros documentos enfatizan ciertos rasgos como relevantes. Entre ellos se destaca la idea de que la EU implica diálogo, intercambio de saberes, lo que plantea la necesidad de una reflexión de índole epistemológica con consecuencias éticas y políticas no menores. En este punto, el trabajo ha retomado aportes del campo de la sociología, fundamentalmente de la sociología de las ausencias y de las emergencias propuestas por Sousa Santos $(2006,2007)$. El autor pone de relieve, entre otras cosas, la importancia del reconocimiento de la diversidad de conocimientos para el logro de la justicia social.

Por otra parte, la EU pensada como diálogo de saberes supone un principio de reconocimiento del otro en su diversidad que debe considerarse desde una perspectiva ética. La noción de ética dialógica (Erausquin, 2013) enfatiza el compromiso e implicación con el destino común de la humanidad que conlleva toda intervención profesional y la búsqueda de consensos que no eludan los conflictos.

En el contexto de este trabajo, la integralidad o prácticas integrales no se reducen a la reflexión relativa al modo en que la EU se relaciona con la enseñanza y la investigación sino que permite delimitar la especificidad de cada una de ellas en el marco de un sistema de relaciones. Este impone en la actualidad un desafío muy importante a la universidad en su conjunto. La noción de sistema de actividad propuesta por Engeström (2001a, 2001b, 2016) ha sido considerada una contribución teórica para el abordaje de esta temática. Los principios fundamentales de la teoría caracterizan a toda actividad humana en tanto sistema, destacando su carácter histórico, su multivocidad, la contradicción como fuente de conflicto y cambio, que puede tener un sentido expansivo cuando se repiensan el objeto y el motivo de la actividad, es decir, qué universidad queremos y para qué.

Para finalizar, cabe señalar que el conjunto de los desarrollos presentados en este trabajo permite vislumbrar la riqueza del camino recorrido por la EU en los últimos años bajo la consigna de su "jerarquización". Tal vez sea tiempo de que la EU reformule aspectos de su agenda, capitalice los logros obtenidos y redefina la jerarquización en un diálogo más abierto con la enseñanza y la investigación. 


\section{Referencias bibliográficas}

Arocena, R. (2013). Prólogo. Curricularización de la extensión: ¿por qué, cuál, cómo? En Arocena, R.; Tommasino, H.; Rodríguez, N.; Sutz, J.; Alvárez Pedrosian, E. y Romano, A. (Eds.). Cuadernos de Extensión Nº 1. Integralidad: tensiones y perspectivas (pp. 9-17). Montevideo: Comisión Sectorial de Extensión y Actividades en el Medio (CSEAM). Recuperado de: http://www.pim.edu.uy/files/2013/05/Cuaderno_integralidad-1.pdf (30/05/2018).

Cecchi, N. (2015). La Universidad que tenemos y la que deberíamos ser: una Universidad socialmente comprometida. En Medina, J.M. (Comp.). Publicación del VI Congreso Nacional de Extensión Universitaria. La Universidad en diálogo con la Comunidad. Construyendo una Institución en contexto (pp. 23-32). Rosario: UNR Editora. Engeström, Y. (2001a). Los estudios evolutivos del trabajo como punto de referencia de la teoría de la actividad: el caso de la práctica médica de la asistencia básica. En Chaiklin S. \& Lave, J. (Comps.). Estudiar las prácticas. Perspectivas sobre actividad y contexto (pp. 78-88). Buenos Aires: Amorrortu.

(2001b). Expansive Learning at Work: Toward an activity theoretical reconceptualization [El aprendizaje expansivo en el trabajo: hacia una reconceptualización teórica de la actividad]. Journal of Education and Work, 14(1), 133-156.

Engeström, Y. y Sannino, A. (2016) El aprendizaje expansivo en movimiento aportaciones de la investigación en curso. Infancia y Aprendizaje: Journal for the Study of Education and Development, 39(3), 417-435.

Erausquin, C. (2013). Ayudando a los que ayudan a aprender: co-construyendo acciones y conocimiento. Ficha de cátedra, Facultad de Psicología, UNLP.

Erausquin, C. y Zabaleta, V. (2014). Articulación entre investigación y extensión universitarias: aprendizajes en la diversidad. EXT. Divulgación y discusión de experiencias, métodos, tecnologías y propuestas teóricas referidas a la Extensión Universitaria, (5). Secretaría de Extensión de la Universidad de Córdoba. Recuperado de: http://revistas.unc.edu.ar/index.php/ext/article/view/7442 (30/05/2018).

Estatuto de la Universidad Nacional de La Plata (2008). Recuperado de: http://www.unlp.edu.ar/uploads/ docs/estatuto_2008_final.pdf (30/05/2018).

Ferrero, A. (2012). Ética y Deontología Profesional en Psicología. Conferencia dictada en Simposio Regional de la Sociedad Interamericana de Psicología. Santa Cruz de la Sierra, Bolivia.

Maiorana, D. (2015). Hablar de diálogo y de contexto es hablar de Extensión. En Medina, J.M. (Comp.). Publicación del VI Congreso Nacional de Extensión Universitaria. La Universidad en diálogo con la Comunidad. Construyendo una Institución en contexto (pp. 11-15). Rosario: UNR Editora.

Menéndez, G. (2015). La integración de las funciones sustantivas y la misión social de la Universidad. En En Medina, J.M. (Comp.). Publicación del VI Congreso Nacional de Extensión Universitaria. La Universidad en diálogo con la Comunidad. Construyendo una Institución en contexto (pp. 32-42). Rosario: UNR Editora.

Menéndez, G. y Tarabella, L. (2016). El aprendizaje experiencial: una práctica de innovación que se afianza en la Universidad Nacional del Litoral. Revista +E versión digital, (6), 96-103. Recuperado de: https://bibliotecavirtual.unl.edu.ar/publicaciones/index.php/Extension/article/view/6317/9283 (30/05/2018).

Plan Estratégico de Gestión (2014-2018). Universidad Nacional de La Plata. Recuperado de: http://www.unlp. edu.ar/plan_estrategicio (30/05/2018).

Sousa Santos, B. de (2006). La Sociología de las Ausencias y la Sociología de las Emergencias: para una ecología de saberes. En Renovar la teoría crítica y reinventar la emancipación social (encuentros en Buenos Aires) (pp. 13-41). Recuperado de: http://bibliotecavirtual.clacso.org.ar/ar/libros/edicion/santos/Capitulo\%20l.pdf

- (2007). La Universidad en el siglo XXI. Para una reforma democrática y emancipatoria de la universidad. La Paz: Plural Editores. 
Sutz, J. (2013). La integralidad de las funciones universitarias como espacio de preguntas recíprocas. En Arocena, R.; Tommasino, H.; Rodríguez, N.; Sutz, J.; Alvárez Pedrosian, E. y Romano, A. (Eds.). Cuadernos de Extensión $N^{\circ}$ 1. Integralidad: tensiones y perspectivas (pp. 43-58). Montevideo: Comisión Sectorial de Extensión y Actividades en el Medio (CSEAM). Recuperado de: http://www.pim.edu.uy/files/2013/05/Cuaderno_integralidad-1.pdf (30/05/2018).

Tauber, F. (2016). Proyecto Institucional de la Universidad Nacional de La Plata (2018-2022). Pensar la Universidad. Recuperado de: http://www.unlp.edu.ar/pensarlaunlp (30/05/2018).

Tommasino, H. y Rodríguez, N. (2013). Tres tesis básicas sobre extensión y prácticas integrales en la Universidad de la República. En En Arocena, R.; Tommasino, H.; Rodríguez, N.; Sutz, J.; Alvárez Pedrosian, E. y Romano, A. (Eds.). Cuadernos de Extensión No 1. Integralidad: tensiones y perspectivas (pp. 19-39). Montevideo: Comisión Sectorial de Extensión y Actividades en el Medio (CSEAM). Recuperado de: http://www.pim.edu.uy/ files/2013/05/Cuaderno_integralidad-1.pdf (30/05/2018). 